\title{
A Quality Assurance Approach for Linear Accelerator Mechanical Isocenters with Portal Images
}

\author{
Qiyong Fan', Sumin Zhou' ${ }^{1}$, Yu Lei' ${ }^{1}$, Sicong Li', Mutian Zhang2* \\ ${ }^{1}$ Department of Radiation Oncology, University of Nebraska Medical Center, Omaha, NE, USA \\ ${ }^{2}$ Summa Health Cancer Institute, Akron, OH, USA \\ Email: *zhangm@summahealth.org
}

How to cite this paper: Fan, Q., Zhou, S., Lei, Y., Li, S. and Zhang, M. (2018) A Quality Assurance Approach for Linear Accelerator Mechanical Isocenters with Portal Images. International Journal of Medical Physics, Clinical Engineering and Radiation Oncology, 7, 100-114.

https://doi.org/10.4236/ijmpcero.2018.71009

Received: January 11, 2018

Accepted: February 24, 2018

Published: February 27, 2018

Copyright $\odot 2018$ by authors and Scientific Research Publishing Inc. This work is licensed under the Creative Commons Attribution International License (CC BY 4.0).

http://creativecommons.org/licenses/by/4.0/

(C) (i) Open Access

\begin{abstract}
Purpose: With usually a millimeter-level PTV margin, stereotactic radiosurgery (SRS) and stereotactic body radiation therapy (SBRT) pose a stringent requirement on the isocentricity of the Linac. This requirement is partly fulfilled by routine isocenter quality assurance (QA) test to verify the size and location of the isocenter. The current common QA methods such as spoke shot were developed before SBRT/SRS became popular and when IGRT was largely absent and hence have their limitations. In this work, we describe an isocenter QA approach based on portal imaging to provide the community with a superior alternative. Methods: The proposed approach utilizes a BrainLab ball bearing (BB) phantom in conjunction with an electronic portal imaging devices (EPID) imager. The BB phantom was first aligned with a calibrated room laser system. Portal images were then acquired using $6 \mathrm{MV}$ beam with a $2 \times 2 \mathrm{~cm}^{2}$ open field and a $15 \mathrm{~mm}$ cone on a Varian TrueBeam STx machine. The gantry, collimator, and table were rotated separately at selected angles to acquire a series of portal images in order to determine the isocenter of each rotating system. The location and diameter of these isocenters were determined by calculating the relative displacement of either $\mathrm{BB}$ or open field edge between the acquired EPID images. The demonstration of the reproducibility and robustness of this EPID-based approach was carried out by repeating measurements 10 times independently for each rotating system and simulating clinical scenarios of asymmetric jaws and misalignment of BB phantom, respectively. Results: For our TrueBeam STx machine, the isocenter diameter derived from open-field EPID images was roughly $0.15 \mathrm{~mm}, 0.18$ $\mathrm{mm}, 0.49 \mathrm{~mm}$ for the collimator, table, and gantry, respectively. For the collimator and gantry, images taken with the cone gave considerably smaller isocenter diameter. Results remained almost unchanged despite the presence of
\end{abstract}


simulated $\mathrm{BB}$ misalignment and asymmetric jaws error, and between independent measurements. Isocenter location and diameter derived from images obtained at a limited number of angles $(\leq 11)$ were adequately accurate to represent those derived from images of densely sampled angles. Conclusions: An EPID-based isocenter QA approach is described and demonstrated to be accurate, robust, and reproducible. This approach provides a superior alternative to conventional isocenter QA methods with no additional cost. It can be implemented with convenience for any linear accelerator with an EPID imager.

\section{Keywords}

Linear Accelerator, Isocenter, Mechanical Check, Quality Assurance, Spoke Shot

\section{Introduction}

With the spread use of image guided radiation therapy (IGRT), stereotactic radiosurgery (SRS) and stereotactic body radiation therapy (SBRT) have become popular options among the eligible patients due to their favorable clinical outcome and logistic convenience of only one or a few treatments [1] [2] [3]. For contemporary linear accelerators (Linacs) that deliver the SRS/SBRT, the mechanical accuracy is increasingly an important issue as those types of treatments usually entail the use of small planning target volume (PTV) margins to account for treatment uncertainties. Therefore, a Linac with inferior mechanical performance, especially with a large and unstable mechanical isocenter, may compromise the effectiveness of the whole treatment workflow. Consequently, quality assurance (QA) checks are routinely performed to ensure the mechanical isocenter accuracy of medical accelerators [4] [5]. For instance, Task Group 142 (TG-142) of the American Association of Physicists in Medicine (AAPM) recommends that the collimator, couch, and the gantry rotation isocenters should be checked against baseline with $\pm 1 \mathrm{~mm}$ tolerance during annual QA [6], which replaces the tolerance of $2 \mathrm{~mm}$ diameter as set forth by the AAPM Task Group 40 (TG-40) [7].

Traditionally, the Linac isocenter diameter is evaluated with "star-shot" or spoke shot patterns formed by collimated narrow radiation fields on radiographic film [8] [9]. Although the spoke shot method is still used by many therapeutic medical physicists, its reliability is often compromised by some pitfalls. For example, the accuracy of spoke shot depends on the accurate calibration of Linac jaws or multileaf collimator. Also, mechanical instability along the narrow field cannot be effectively detected. Moreover, it is rather difficult to quantify the coincidence of different isocenters in three-dimensional (3D) space using spoke shot. Finally, the measurement setup and film analysis may lead to additional complexity and uncertainty in determining the isocenter diameter.

Other Linac isocenter QA techniques have also been developed [4]. One pioneering work was done by Lutz et al., who developed an apparatus to test the 
coincidence of collimator and gantry rotation axes [10]. This apparatus was attached to the collimator or gantry, and could punch semi-circle or full-circle marks on a cardboard during collimator or gantry rotation. The two centers constructed from the marks were used to check the coincidence of the collimator and gantry isocenters. Later, there were implementations of utilizing a ball bearing (BB) to evaluate the isocentricity and help improve the practice of Linac isocenter QA. Winston-Lutz test is one such category of methods and undoubtedly, is the most popular QA test nowadays that examines the target position with respect to collimated radiation fields prior to the SRS/SBRT procedures [11]. However, the Winston-Lutz test based on radiographic film cannot give the values of rotation isocenter diameter and commercial software such as DoseLab Pro (Mobius Medical Systems, Houston, TX, USA) is often needed for image analysis [12]. More importantly, neither spoke-shot nor Winston-Lutz test can give the 3D location of the isocenter of each rotation component, which is essential for comparison against the baseline and evaluation of the isocenter stability with respect to a stationary reference coordinate system such as the Linac vault.

In this work, we describe a detailed implementation of a QA approach for Linac mechanical isocenter which can analyze both the diameter and the 3D location of the isocenters. This approach is based on the readily available image guidance provided by the contemporary Linacs. Although it is known that image guidance can greatly reduce treatment uncertainties such as imperfect patient setup, possible patient weight loss, and physiologic motion, an often overlooked application of the image guidance in radiation therapy is its use for the QA of the medical linear accelerator. Hence, this work intends to further exploit the benefits of such image guidance in this aspect. Specifically, we take advantage of the fact that contemporary medical Linacs are commonly equipped with amorphous silicon flat-panel detectors with sub-millimeter pixel size. These electronic portal imaging devices (EPID) can take high spatial resolution MV portal images using low monitor units (MUs) of radiation [13] [14]. In fact, there is previous work that has demonstrated the EPID allows the detection of a $\mathrm{BB}$ centroid position at ultra-high accuracy $(\sim 0.01 \mathrm{~mm})[15]$.

With high resolution EPID images, it is feasible to determine the isocenter diameter and spatial location based on our previously published theoretical framework of isocenter definition [16]. According to the proposed framework, the Linac gantry, collimator, and table systems are considered rigid bodies, and their isocenters are defined using the axis of rotation (AOR) observed in a stationary reference system. The AOR is determined by the full trajectory of a point on the rigid body that has the minimal bounding sphere (or circle in the $2 \mathrm{D}$ case). In order to implement the above definition in this study, a BB phantom is placed in proximity of the isocenter to be measured, and a series of MV portal images are taken for selected angles of each rotational component (gantry, collimator, or table). These images should use the same jaw settings or the same circular cone, so that the relative displacement of either BB or open field edge between selected 
rotation angles can be detected through image analysis. Based on these results, an in-house software package calculates the $3 \mathrm{D}$ location and size of the isocenters. The proposed method provides the medical physics community with a convenient and accurate alternative for the Linac mechanical isocenter QA.

\section{Methods}

\subsection{Image Acquisition and Analysis}

All the portal images were acquired using the MV imager on a TrueBeam STx Linac (Varian Medical Systems, Palo Alto, CA, USA) at $150 \mathrm{~cm}$ source to image distance. When projected at the Linac isocenter, the imager pixel size is 0.26 mm. A BB phantom (BrainLab AG, Feldkirchen, Germany) was secured on the cranial end of the table. At the beginning of the imaging process, the gantry, collimator, and the table were set at $0^{\circ}$ angles, and the $\mathrm{BB}$ was aligned to the room lasers (LAP Laser, Lüneburg, Germany). These lasers were adjusted using a flexmap approach so that they could closely represent the radiation isocenter [13] [17]. High resolution single-exposure images of the BB phantom both in jaw-defined and circular cone-defined fields were taken using $3 \mathrm{MU}$ of $6 \mathrm{MV}$ beam. The field size was $2 \times 2 \mathrm{~cm}^{2}$ (jaw) and $15 \mathrm{~mm}$ (cone), respectively. The images were processed and analyzed using MATLAB (The Math Works, Inc., Natick, MA, USA). Throughout this study, the $x, y, z$ coordinates follow the IEC 61217 convention.

Spoke shot patterns were derived on the Oncology EDR2 films (Carestream Health Inc., Rochester, NY, USA) sandwiched between Solid Water (Gammex Inc., Middleton, WI, USA) slabs and placed at the Linac isocenter level. In this study, only one rotating system (collimator) was illustrated for comparison purpose. At each collimator angle, 100-120 MU of $6 \mathrm{MV}$ beam was delivered. The processed films were scanned using a Dosimetry Pro Advantage film scanner (Vidar Systems Corporation, Herndon, VA, USA), and the spoke shot pattern was analyzed using DoseLab Pro to determine the isocenter size in the $2 \mathrm{D}$ plane.

\subsection{Determination of Rotation Isocenters}

\subsubsection{Collimator and Table Rotation Isocenter}

The workflow to determine the collimator isocenter is depicted in Figure 1. As shown, the isocenter is determined by calculating the relative shifts of the fields between that at $0^{\circ}$ and those at other selected collimator angles (i.e., $40^{\circ}, 80^{\circ}$, $120^{\circ}, 160^{\circ}, 200^{\circ}, 240^{\circ}, 280^{\circ}, 320^{\circ}$ in this study). This was achieved by first extracting the field edge using the classical Canny algorithm with a threshold of 0.7 (for jaw/cone edge only) and 0.3 (for both jaw/cone edge and BB contour) for all the collimator angles. Note that the same parameters of edge detection were used throughout this study. A mask was then generated by filling a constant value of 1 inside the field edge and a weighted centroid was computed for each mask to represent its location. Subsequently, a rough collimator isocenter was calculated 


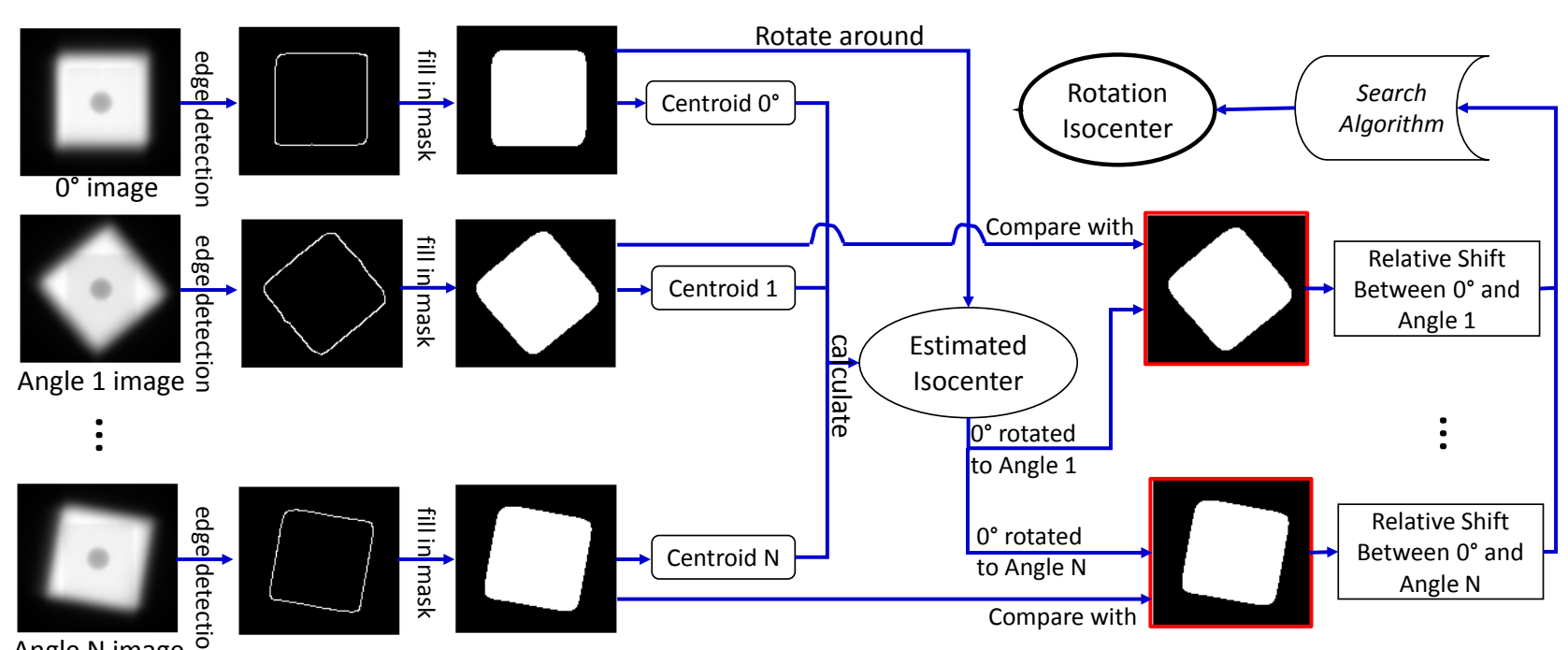

Angle $\mathrm{N}$ image $\stackrel{\overline{\mathrm{o}}}{\mathrm{s}}$

Figure 1. The workflow to determine the collimator isocenter. Field edges were first extracted from each image based on the classical Canny algorithm with a fixed threshold. A mask was then generated by filling a constant value of 1 inside the field edge. The estimated isocenter was calculated as the centroid of the individual masks' centroids. The $0^{\circ}$ mask was subsequently rotated around the estimated isocenter to other angles in order to be compared with the masks of those angles. Relative shifts were calculated between $0^{\circ}$ mask and the masks of other angles. These shifts were finally utilized to determine the collimator isocenter via the search algorithm as described in Section 2.2.

as the centroid of the nine mask-centroids. In this way, the relative shift between the zero-degree field edge and the field edge at other angles can be determined by rotating the zero-degree field edge to other angles around the rough collimator isocenter. The estimation uncertainty introduced by the rough isocenter not being the real isocenter during this process is assumed minimal. Finally, the isocenter was determined by literally implementing the isocenter definition according to a previously published paper [16], i.e., to search a point within the field edge whose trajectory of rotation gives a minimum bounding circle given the relative shifts between all the rotations. This was achieved by a two-step brute-force searching algorithm [16]. The first step searched the approximate location of such point using a large search step size and then the second step continued to conduct the search near the approximate location with a much smaller search step size of $0.01 \mathrm{~mm}$. During each search step, the exact minimum bounding circle of a trajectory was computed using the Welzl's algorithm [18]. After the search, the resultant point with minimum bounding circle represented the AOR of the rotating system, and the center of the smallest enclosing circle was regarded as the projection of the isocenter of this system while its radius represented the size of the isocenter. The same workflow has been applied for the determination of the table isocenter except that not the mask of the field edge but the actual image of $\mathrm{BB}$ was used to calculate the relative shifts. This is due to the fact that as the table rotates, the $\mathrm{BB}$ moves but the field edge stays stationary, opposite to the collimator case. The selected table rotation angles are $0^{\circ}$, $15^{\circ}, 35^{\circ}, 55^{\circ}, 75^{\circ}, 95^{\circ}, 265^{\circ}, 285^{\circ}, 305^{\circ}, 325^{\circ}, 345^{\circ}$.

The underlying principle of the above method is that the collimator and the 
table are treated as rigid bodies. When observed in a stationary reference system, e.g., the Linac vault, the AOR of a rigid body tends to wiggle while it rotates. Because all the points on the rigid body have identical angular velocity relative to each other, the AOR has to be defined within the stationary reference system using the concept of the smallest enclosing sphere/circle, as suggested by the previous investigators [19] [20]. For detailed discussion on the isocenter definition of the collimator and table, please refer to the previously published work [16].

\subsubsection{The Gantry Rotation Isocenter}

Unlike the collimator and table where 2D analysis was sufficient for QA purpose, the gantry rotation isocenter was analyzed in the 3D space. In this study, the variations in $\mathrm{x}$-ray source position and gantry angle were assumed to be negligible for simplification. The position of the open field center relative to the $\mathrm{BB}$ center was regarded as a surrogate of the gantry mechanical instability at a given gantry angle. In the 3D space, straight lines passing through the $\mathrm{x}$-ray source and field center were plotted for gantry angles at which the images were taken. The gantry rotation isocenter was defined as the center of the smallest sphere through whose surface all the above straight lines passing. This intuitive definition is an approximation of what was previously proposed [16]. The actual implementation of such definition is shown in Figure 2.

As illustrated in Figure 2, we first defined a search cube of $1 \times 1 \times 1 \mathrm{~mm}^{3}$ around the center of the $\mathrm{BB}$ phantom at $0^{\circ}$ gantry angle, which was assumed to

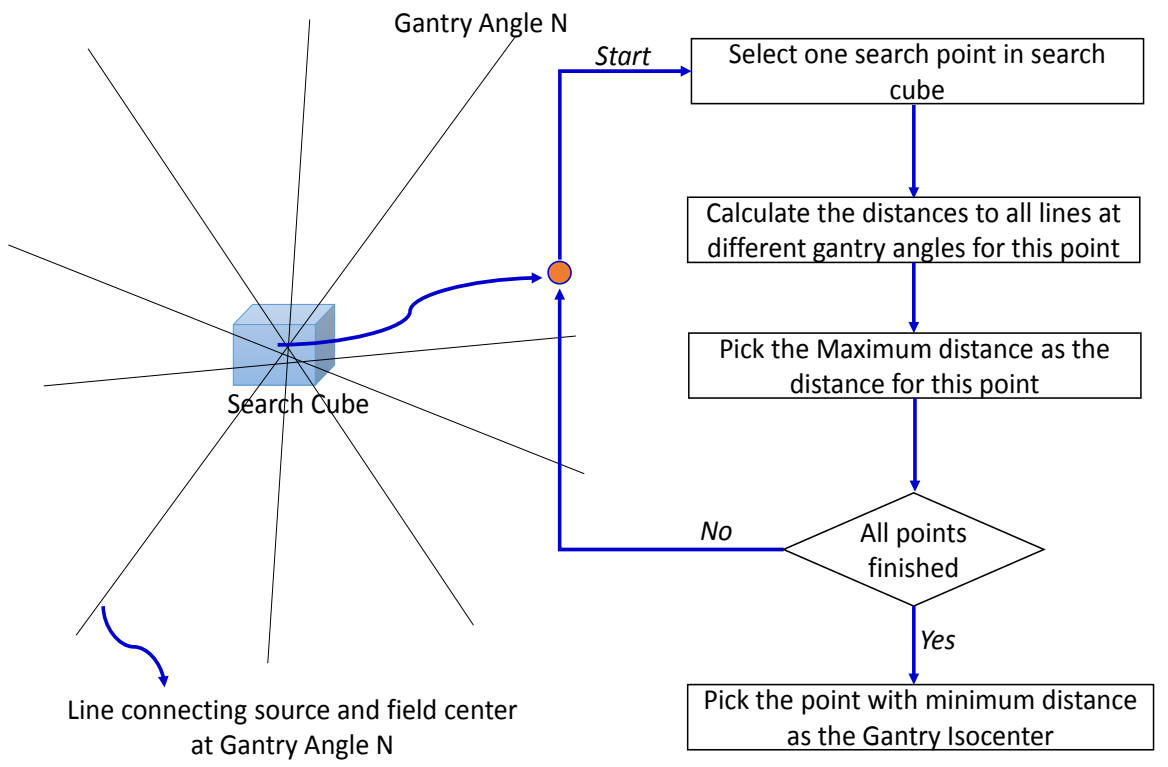

Figure 2. The workflow to determine the rotation isocenter of gantry. A search cube of 1 $\mathrm{mm}^{3}$ was defined around the center of the BB phantom at $0^{\circ}$ gantry angle. Points were then sampled within this cube and distances from each sampled point to the lines that connect the source and the field center at all the given gantry angles. The maximum distance of the distances to all the straight lines was taken as the distance for each individual point. The sampled point that had the minimum distance was designated as the isocenter. 
contain the gantry isocenter (most contemporary Linacs satisfy this requirement). Then we sampled points within this cube with a fine resolution of 0.01 $\mathrm{mm}$ and calculated the distances from each individual point to the lines that connect the source and the field center at all the given gantry angles. We designated the maximum distance of the distances to all the straight lines as the distance for each individual point. The point that had the minimum distance was taken as the isocenter according to the aforementioned gantry isocenter definition.

\subsection{Reproducibility and Robustness of the Proposed QA Approach}

The reproducibility of the proposed new QA approach was tested using repeated imaging measurements. The BB was aligned to the room lasers, and a series of portal images were taken at selected angles of one rotating system (collimator, gantry, or table) using jaw-defined $2 \times 2 \mathrm{~cm}^{2}$ open field, while the other two systems remained at the $0^{\circ}$ angle. For each rotating component, this process was independently repeated 10 times with the $\mathrm{BB}$ position unchanged.

The robustness of the proposed approach was demonstrated by intentionally changing the $\mathrm{BB}$ position and using asymmetric radiation field. For illustration purpose, only the demonstration for the collimator isocenter was performed. In detail, the BB phantom was first aligned to the lasers. Images were then taken at nine selected collimator angles similarly as before using jaw-defined $2 \times 2 \mathrm{~cm}^{2}$ open fields and with gantry and couch angle at $0^{\circ}$ under two separate scenarios: 1) the $\mathrm{BB}$ phantom remained at the same position, but each of the four jaws was opened in turn by $2 \mathrm{~mm}$ to form an asymmetric field. Images were taken for each asymmetric field for nine collimator angles; 2 ) the BB phantom was shifted in turn toward four directions (left and right, in and out) by $1 \mathrm{~mm}$ from its original position, and images were taken for each of the four positions at nine collimator angles.

For comparison, the performance of the spoke shot method in the presence of asymmetric jaw was evaluated on the same TrueBeam Linac using the radiographic films. Spoke shot images were taken at nine collimator angles similarly with different X-jaw slot offsets of $0, \pm 0.5 \mathrm{~mm}$, and $\pm 1.0 \mathrm{~mm}$ from the nominal collimator center. Positive offsets were towards the patient left, or the X2 jaw direction. The films were scanned and analyzed as described in Section 2.1.

\subsection{Adequacy Study}

The proposed approach aims to determine the isocenter for each rotating system using only a limited number of images $(\leq 11)$. In order to investigate whether these images were adequate for accurately determining the isocenters, an adequacy study was devised to compare the above results with those derived from much densely sampled portal images. Specifically, the BB images were acquired using jaw-defined $2 \times 2 \mathrm{~cm}^{2}$ open fields at every $5^{\circ}$ within the possible rotation 
ranges of the gantry, collimator, and table, respectively. The isocenter location and size of each component were calculated with the whole image set, and with a selected subset of these images corresponding to the rotation angles as detailed in Section 2.2.

\section{Results}

\subsection{Isocenter Determination}

Figures 3-5 depict the isocenter determination results for collimator, table, and gantry, respectively. Figure 3(a) shows both the trajectory of the point that gave the minimum bounding circle (called "Mini-circle Point") and the resulted minimum bounding circle for the case where 9 jaw-defined EPID images were used. Figure 3(b) describes the same results as Figure 3(a) except the EPID

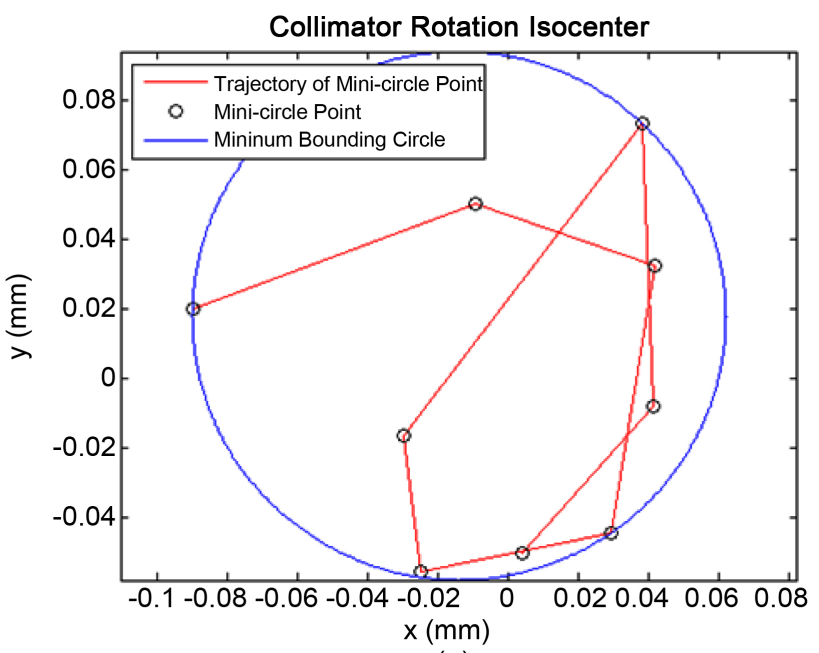

(a)

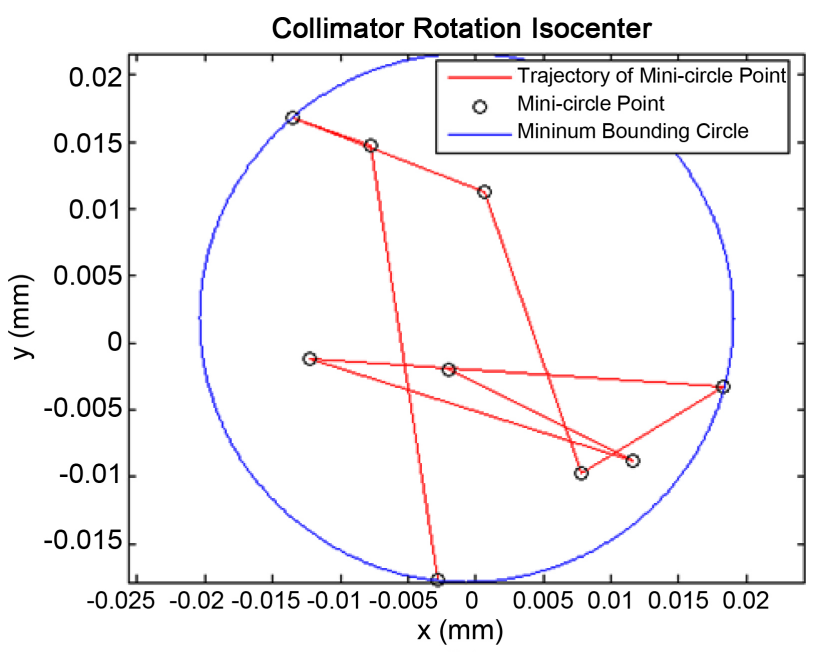

(b)

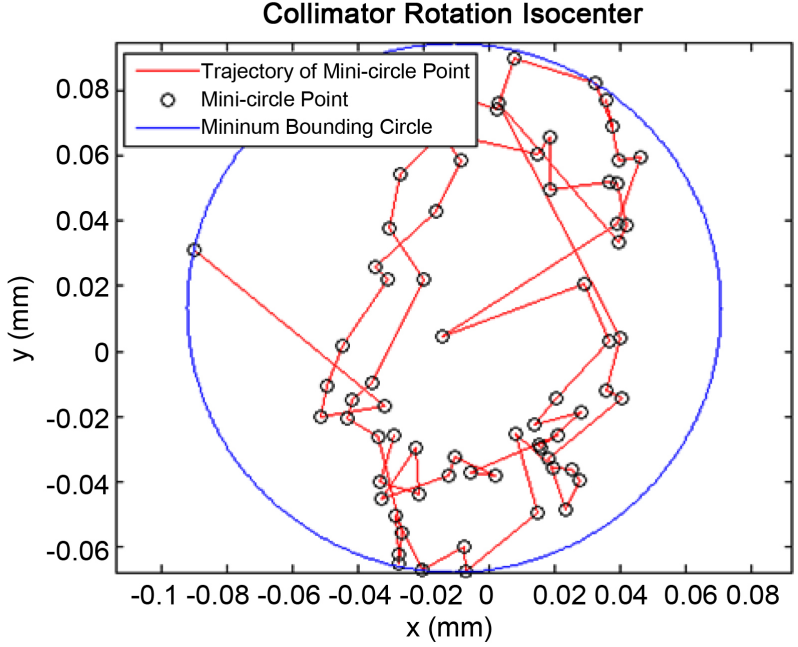

(c)

Figure 3. Determination of the collimator rotation isocenter for (a) jaw-defined field with 9 images, (b) cone-defined field with 9 images, and (c) jaw-defined field with 71 images. The coordinates of the collimator isocenter relative to the radiation isocenter are $(-0.02,0.02) \mathrm{mm},(-0.00,0.00) \mathrm{mm}$, and $(-0.01,0.01) \mathrm{mm}$, respectively. The diameter of the three mechanical isocenters were $0.15 \mathrm{~mm}, 0.04 \mathrm{~mm}$, and $0.16 \mathrm{~mm}$, respectively. 


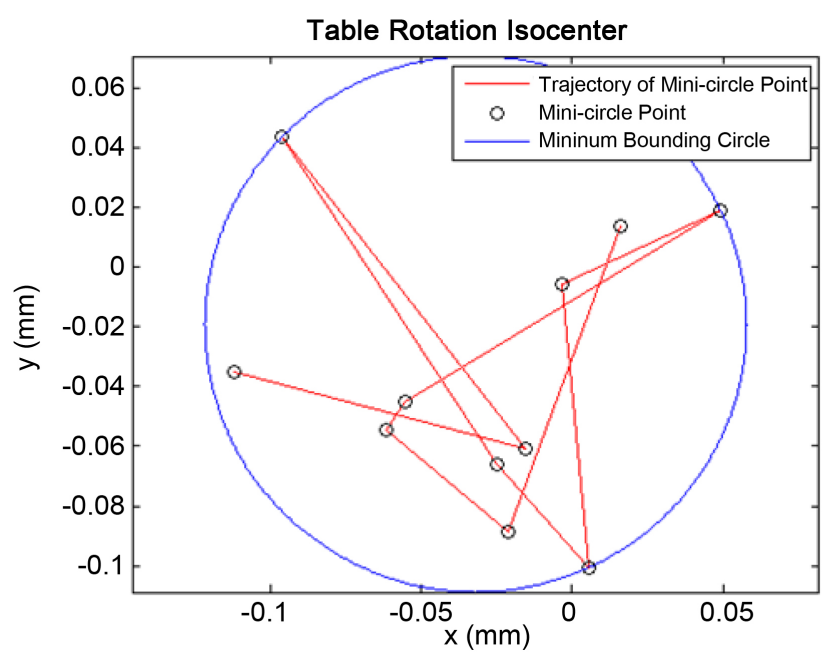

(a)

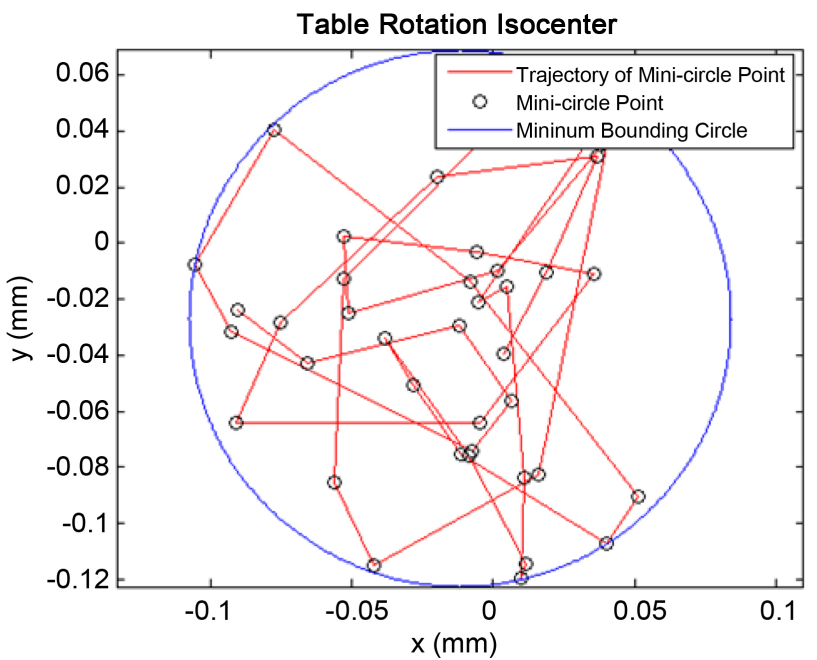

(c)

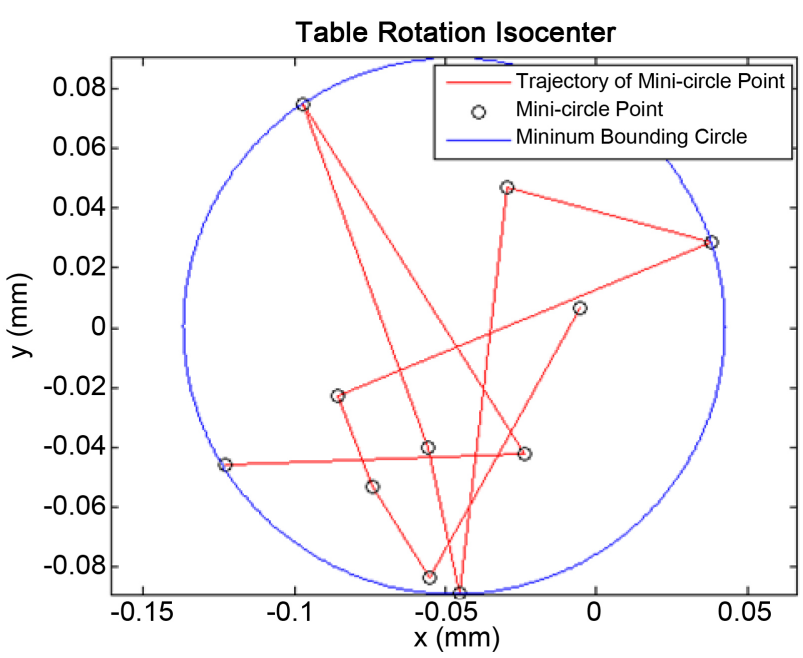

(b)

Figure 4. Determination of the table rotation isocenter for (a) jaw-defined field with 11 images, (b) cone-defined field with 11 images, and (c) jaw-defined field with 39 images. The coordinates of the table isocenter relative to the radiation isocenter are $(-0.03,0.02) \mathrm{mm},(-0.05,0.00) \mathrm{mm}$, and $(-0.01,0.03) \mathrm{mm}$, respectively. The diameter of the three mechanical isocenters were $0.18 \mathrm{~mm}, 0.18 \mathrm{~mm}$, and $0.19 \mathrm{~mm}$, respectively.

images were acquired with cone-defined field. Figure $3(\mathrm{c})$ presents the trajectory and the minimum bounding circle with 71 EPID images (densely sampled along the rotation range of collimator). The coordinates of the collimator isocenter relative to the radiation isocenter were $(-0.02,0.02) \mathrm{mm},(-0.00,0.00)$ $\mathrm{mm}$, and $(-0.01,0.01) \mathrm{mm}$, respectively. The diameter of the three mechanical isocenters were $0.15 \mathrm{~mm}, 0.04 \mathrm{~mm}$, and $0.16 \mathrm{~mm}$, respectively.

Figure 4 shows the counterpart results of Figure 3 for table rotation, except 11 and 39 EPID images were used for Figure 4(a) and Figure 4(c), respectively. As a result, the coordinates of the table isocenter relative to the radiation isocenter were $(-0.03,0.02) \mathrm{mm},(-0.05,0.00) \mathrm{mm}$, and $(-0.01,0.03) \mathrm{mm}$, respectively. The diameter of the three mechanical isocenters were $0.18 \mathrm{~mm}, 0.18 \mathrm{~mm}$, and $0.19 \mathrm{~mm}$, respectively. 


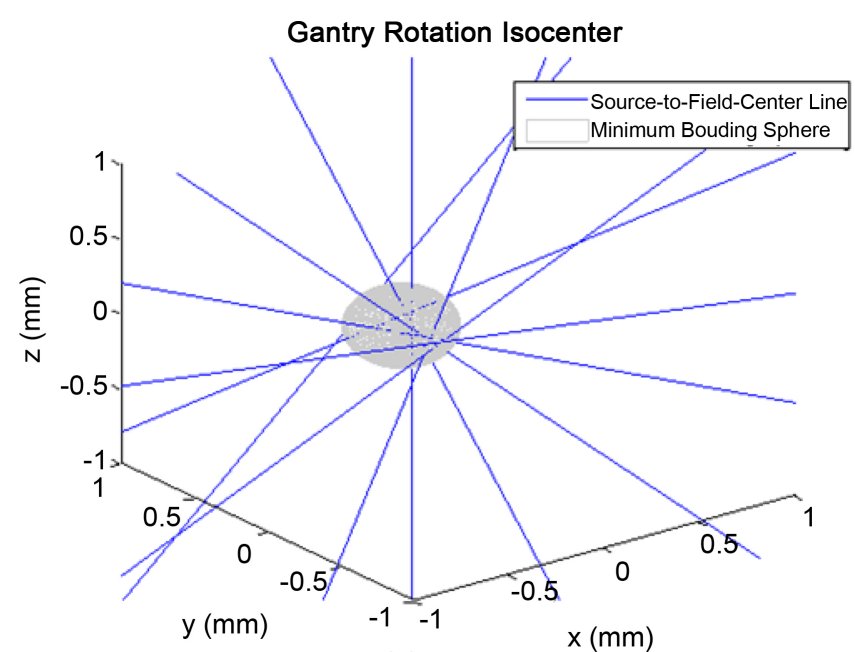

(a)

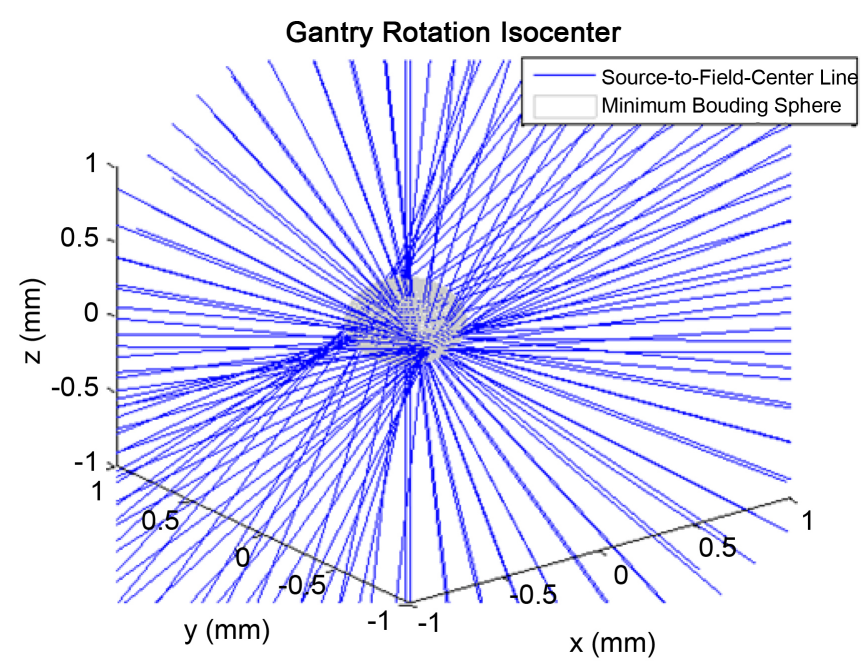

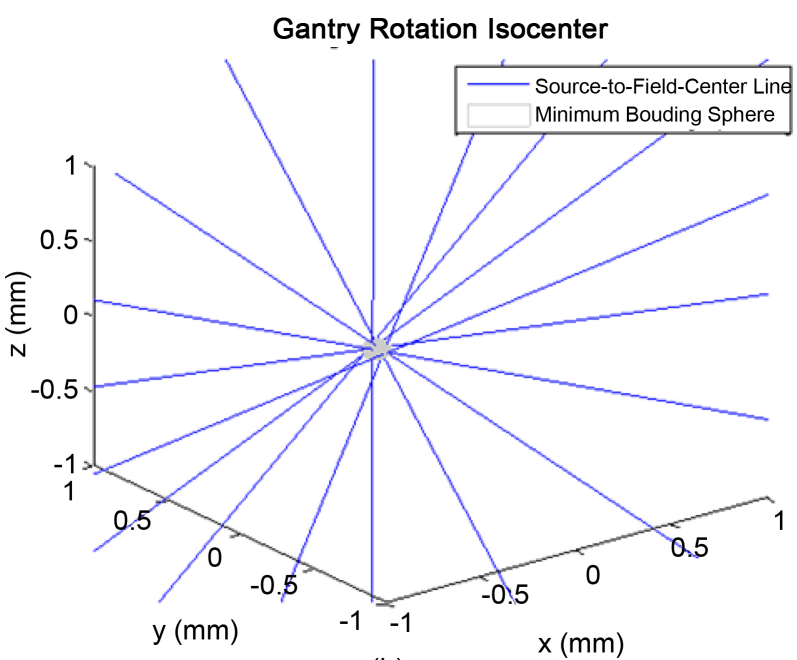

(b)

(c)

Figure 5. Determination of the gantry rotation isocenter for (a) jaw-defined field with 9 images, (b) cone-defined field with 9 images, and (c) jaw-defined field with 72 images. The coordinates of the gantry isocenter relative to the radiation isocenter are $(-0.23,0.09,0.06) \mathrm{mm},(-0.28,0.01,-0.03) \mathrm{mm}$, and $(-0.19,0.08,0.06) \mathrm{mm}$, respectively. The diameter of the three mechanical isocenters were $0.49 \mathrm{~mm}, 0.12 \mathrm{~mm}$, and $0.50 \mathrm{~mm}$, respectively.

Figure 5 demonstrates the gantry rotation isocenter results in 3D space. Each sub-figure shows the lines that connect the source and the center of field (called "Source-to-Field-Center Line") and the minimum bounding sphere that contains those lines. Similarly, Figure 5(a) shows the results using 9 jaw-defined EPID images while Figure 5(b) presents for the case of 9 cone-defined EPID images. Figure 5(c) presents the minimum bounding sphere with 72 EPID images (densely sampled along the gantry rotation circle). The coordinates of the gantry isocenter relative to the radiation isocenter were $(-0.23,0.09,0.06) \mathrm{mm}$, $(-0.28,0.01,-0.03) \mathrm{mm}$, and $(-0.19,0.08,0.06) \mathrm{mm}$, respectively. The diameter of the three mechanical isocenters were $0.49 \mathrm{~mm}, 0.12 \mathrm{~mm}$, and $0.50 \mathrm{~mm}$, respectively. 


\subsection{Reproducibility and Robustness}

Tables 1(a)-(c) summarize the reproducibility of the proposed algorithm for the collimator, table, and gantry, respectively. Each table shows the results of 10 independent image sets for each rotational system. As shown, the standard deviation (SD) for each case is almost negligible, which demonstrates the superior reproducibility of the method.

Table 2 lists the results of our algorithm in the presence of asymmetric fields or shifted BB positions. Despite the intentionally introduced errors of jaw position or BB setup, the analyzed results align well with the ones without the simulated errors. This demonstrates our method is robust even if certain jaw calibration error or BB setup inaccuracy is present.

Table 1. (a) The reproducibility results of collimator isocenter measured with jaw-defined portal images. Unit: $\mathrm{mm}$. (b) The reproducibility results of table isocenter measured with jaw-defined portal images. Unit: $\mathrm{mm}$. (c) The reproducibility results of gantry isocenter measured with jaw-defined portal images. Unit: $\mathrm{mm}$.

(a)

\begin{tabular}{cccccccccccc}
\hline Image set & 1 & 2 & 3 & 4 & 5 & 6 & 7 & 8 & 9 & 10 & SD \\
\hline Center x & -0.01 & -0.00 & -0.01 & -0.00 & -0.01 & -0.00 & -0.01 & -0.00 & -0.01 & -0.00 & $<0.01$ \\
Center y & 0.01 & 0.01 & 0.01 & 0.00 & 0.01 & 0.01 & 0.01 & 0.00 & 0.01 & 0.00 & $<0.01$ \\
Diameter & 0.14 & 0.14 & 0.14 & 0.14 & 0.14 & 0.14 & 0.12 & 0.14 & 0.12 & 0.14 & $<0.01$ \\
\hline
\end{tabular}

(b)

\begin{tabular}{cccccccccccc}
\hline Image set & 1 & 2 & 3 & 4 & 5 & 6 & 7 & 8 & 9 & 10 & SD \\
\hline Center x & -0.06 & -0.06 & -0.07 & -0.08 & -0.08 & -0.07 & -0.06 & -0.06 & -0.03 & -0.01 & 0.02 \\
Center y & -0.04 & -0.03 & -0.05 & -0.04 & -0.05 & -0.05 & -0.04 & -0.04 & -0.04 & -0.06 & 0.01 \\
Diameter & 0.16 & 0.16 & 0.16 & 0.20 & 0.18 & 0.18 & 0.16 & 0.20 & 0.20 & 0.20 & 0.02 \\
\hline
\end{tabular}

(c)

\begin{tabular}{lccccccccccc}
\hline Image set & 1 & 2 & 3 & 4 & 5 & 6 & 7 & 8 & 9 & 10 & SD \\
\hline Center x & -0.32 & -0.27 & -0.27 & -0.30 & -0.31 & -0.28 & -0.31 & -0.21 & -0.25 & -0.32 & 0.04 \\
Center y & 0.04 & 0.04 & 0.04 & 0.04 & 0.04 & 0.04 & 0.04 & 0.03 & 0.03 & 0.04 & $<0.01$ \\
Center z & 0.07 & 0.06 & 0.05 & 0.07 & 0.05 & 0.06 & 0.08 & 0.13 & 0.08 & 0.07 & 0.02 \\
Diameter & 0.50 & 0.50 & 0.52 & 0.52 & 0.52 & 0.50 & 0.50 & 0.50 & 0.50 & 0.52 & $<0.01$ \\
\hline
\end{tabular}

Table 2. Collimator isocenter coordinates and diameter measured from asymmetric fields or shifted BB positions. As a comparison, the average values from Table 1 (a) are -0.01 , 0.01 , and 0.14 , respectively. Unit: $\mathrm{mm}$.

\begin{tabular}{ccccccccc}
\hline & X1_2mm & X2_2mm & Y1_2mm & Y2_2mm & In_1mm & Out_1mm & Left_1mm & Right_1mm \\
\hline Center x & -0.00 & 0.00 & -0.00 & -0.01 & -0.01 & -0.00 & 0.00 & -0.00 \\
Center y & 0.01 & -0.01 & 0.00 & 0.01 & 0.01 & 0.01 & 0.00 & 0.00 \\
Diameter & 0.20 & 0.12 & 0.16 & 0.14 & 0.12 & 0.12 & 0.16 & 0.14 \\
\hline
\end{tabular}




\subsection{Adequacy}

Comparing Figure 3(a) and Figure 3(c), it is evident that the collimator isocenter results based on 9 EPID images can represent those of 71 images. This is the same case for both table (Figure 4(a) vs Figure 4(c)) and gantry (Figure 5(a) vs Figure $5(\mathrm{c})$ ). In other words, our proposed approach ensures that images from a limited selection of angles, as those listed in Section 2.2., are adequate for the determination of isocenter for each rotational system. This warrants the use of the proposed approach for routine QA.

\subsection{Comparison with Spoke Shot Method}

The spoke shot film results are presented in Table 3 and it shows that, with artificial jaw calibration errors, the collimator isocenter diameter increased rapidly with the jaw offset. The $\pm 1.0 \mathrm{~mm}$ offsets simulated the current tolerance of asymmetric jaw positions [6] [7], and it is clear that jaw calibration errors approaching this tolerance may lead to roughly $2 \mathrm{~mm}$ isocenter diameter estimation error, which would likely cause the spoke shot test to fail [7].

\section{Discussion}

The isocenter QA approach has not substantially evolved as technologies advanced. Developed in the absence of advanced image guidance devices, the spoke shot based techniques inevitably have limitations in the era of IGRT. For the Linac with SRS capability, its inherent uncertainties might be comparable to or even larger than the magnitude of the quantities it intends to measure. In light of the stringent requirement of machine isocentricity posed by SBRT/SRS, this work described in detail a method to directly utilize EPID imaging for isocenter QA and demonstrated an isocenter search algorithm that is accurate, robust, and reproducible. Similar technique has been implemented by Varian Medical System (i.e., IsoLock software) to verify the Linac isocentricity during machine installation [21]. However, the software is proprietary and not available for clinical use, and its algorithm requires a large number of EPID images, making it inconvenient for routine QA purpose. On the contrary, our proposed method only requires a limited number of portal images to verify the isocenter size and location for Linacs equipped with EPID. There are also other attempts to assess the Linac mechanical and radiation isocenters using EPID images, although different advantages exist [22] [23]. For example, Nyflot et al. used an EPID to take $\mathrm{MV}$ images of a $\mathrm{BB}$ mounted on Linac table and aligned to the radiation isocenter [24]. When the table was rotated, the BB trajectory roughly formed a semicircle, which was subsequently fitted to a circle. The center of the fitted circle

Table 3. The effects of jaw calibration error on the size of collimator isocenter measured from spoke shot patterns. Unit: $\mathrm{mm}$.

\begin{tabular}{cccccc}
\hline Jaw offset & -1.0 & -0.5 & 0 & 0.5 & 1.0 \\
$\begin{array}{c}\text { Collimator isocenter } \\
\text { diameter }\end{array}$ & 1.73 & 0.52 & 0.12 & 1.43 & 2.27 \\
\hline
\end{tabular}


was taken as the table rotation isocenter, which could be located relative to the radiation isocenter (i.e., the $\mathrm{BB}$ position at $0^{\circ}$ table angle). However, this method tends to overestimate the size of the isocenter.

In order to minimize the phantom movement and facilitate the image analysis, the $\mathrm{BB}$ phantom must be sufficiently secured on the table. The lateral and vertical movement of the portal imager caused by gantry rotation was assumed minimal. The mechanical instabilities of the jaws can cause certain uncertainties, especially for the case of gantry rotation where gravity tends to change jaw positions and this in turn changes the center of radiation fields. It is therefore recommended that the circular cones are used whenever possible to remove the effects of gravity and ensure reliable results.

The $\mathrm{BB}$ phantom was aligned to the lasers at $0^{\circ}$ collimator/table/gantry angle prior to image collection for each rotational system. The three image sets acquired by rotating the gantry, collimator, or table share one common image, i.e., when the angles of the gantry, collimator, and table were all set at $0^{\circ}$. This image can serve to link the rotational isocenter of each individual rotational system. In other words, one could plot the absolute positions of the gantry, collimator, and table isocenters in the 3D space and evaluate their coincidence with the radiation isocenter by referencing to the above common image.

In addition, despite that our studies are carried out using EPID images, it is worth pointing out that our proposed approach is also valuable to the machines without EPID because the algorithm itself can be implemented likewise on the scanned film images. This was demonstrated to be feasible for a relatively old Linac Primus 1 (Siemens, Germany) during our recent annual QA, when the spoke shot test failed but the open-field film results showed that the table isocenter was within the TG-40 tolerance.

The proposed open field approach is easy to implement in any working environments, as long as the appropriate equipment is in place. It has been successfully used for our TrueBeam STx Linac in the past two years. A user only needs access to a software package that is able to analyze the portal images and determine the isocenters. Once established, this approach should take less time and efforts to complete as required by the conventional spoke shot. The benefits of using this approach include high accuracy, excellent reproducibility, 3D isocenter coincidence check capable, and independent of jaw calibration error. Furthermore, the user can avoid the cost of films, a film processor, and a film scanner if an EPID is available.

\section{Conclusion}

A systematic Linac isocenter quality assurance approach has been developed based on the recently proposed theory of Linac isocenter definition. This approach utilizes portal images of a ball bearing phantom in open fields, and the mechanical isocenters can be measured with respect to a reference point with high accuracy and reproducibility. The method removes the uncertainties asso- 
ciated with jaw calibration and the resultant discrepancies, and is a convenient and powerful alternative to the conventional spoke shot test for Linac mechanical checks.

\section{References}

[1] Timmerman, R., Paulus, R., Galvin, J., et al. (2010) Stereotactic Body Radiation Therapy for Inoperable Early Stage Lung Cancer. JAMA, 303, 1070-1076. https://doi.org/10.1001/jama.2010.261

[2] Andrews, D.W., Scott, C.B., Sperduto, P.W., et al. (2004) Whole Brain Radiation Therapy with or without Stereotactic Radiosurgery Boost for Patients with One to Three Brain Metastases: Phase III Results of the RTOG 9508 Randomised Trial. The Lancet, 363, 1665-1672. https://doi.org/10.1016/S0140-6736(04)16250-8

[3] Zelefsky, M.J., Kollmeier, M., Cox, B., et al. (2012) Improved Clinical Outcomes with High-Dose Image Guided Radiotherapy Compared with Non-IGRT for the Treatment of Clinically Localized Prostate Cancer. International Journal of Radiation Oncology ${ }^{\star}$ Biology $^{\star}$ Physics, 84, 125-129. https://doi.org/10.1016/j.ijrobp.2011.11.047

[4] Rowshanfarzad, P., Sabet, M., O’Connor, D.J. and Greer, P.B. (2011) Isocenter Verification for Linac-Based Stereotactic Radiation Therapy: Review of Principles and Techniques. Journal of Applied Clinical Medical Physics, 12, 185-195. https://doi.org/10.1120/jacmp.v12i4.3645

[5] Gonzalez, A., Castro, I. and Martınez, J. (2004) A Procedure to Determine the Radiation Isocenter Size in a Linear Accelerator. Medical Physics, 31, 1489-1493. https://doi.org/10.1118/1.1755491

[6] Klein, E.E., Hanley, J., Bayouth, J., et al. (2009) Task Group 142 Report: Quality Assurance of Medical Accelerators. Medical Physics, 36, 4197-4212.

https://doi.org/10.1118/1.3190392

[7] Kutcher, G.J., Coia, L., Gillin, M., et al. (1994) Comprehensive QA for Radiation Oncology: Report of AAPM Radiation Therapy Committee Task Group 40. Medical Physics, 21, 581-618. https://doi.org/10.1118/1.597316

[8] Woo, M.K. (2002) A Personal-Computer-Based Method to Obtain "Star-Shots" of Mechanical and Optical Isocenters for Gantry Rotation of Linear Accelerators. Medical Physics, 29, 2753-2755. https://doi.org/10.1118/1.1521937

[9] Depuydt, T., Penne, R., Verellen, D., et al. (2012) Computer-Aided Analysis of Star Shot Films for High-Accuracy Radiation Therapy Treatment Units. Physics in Medicine \& Biology, 57, 2997-3011. https://doi.org/10.1088/0031-9155/57/10/2997

[10] Lutz, W.R., Larsen, R.D. and Bjarngard, B.E. (1981) Beam Alignment Tests for Therapy Accelerators. International Journal of Radiation Oncology ${ }^{\star}$ Biology ${ }^{\star}$ Physics, 7, 1727-1731. https://doi.org/10.1016/0360-3016(81)90201-7

[11] Lutz, W., Winston, K.R. and Maleki, N. (1988) A System for Stereotactic Radiosurgery with a Linear Accelerator. International Journal of Radiation Oncology ${ }^{\star}$ Biology $^{*}$ Physics, 14, 373-381. https://doi.org/10.1016/0360-3016(88)90446-4

[12] Denton, T.R., Shields, L.B., Howe, J.N. and Spalding, A.C. (2015) Quantifying Isocenter Measurements to Establish Clinically Meaningful Thresholds. Journal of Applied Clinical Medical Physics, 16, 175-188. https://doi.org/10.1120/jacmp.v16i2.5183

[13] Imae, T., Haga, A., Saotome, N., et al. (2014) Winston-Lutz Test and Acquisition of Flexmap Using Rotational Irradiation. Japanese Journal of Radiological Technology, 
70, 359-368. https://doi.org/10.6009/jjrt.2014_JSRT_70.4.359

[14] Ravindran, P.B. (2016) A Study of Winston-Lutz Test on Two Different Electronic Portal Imaging Devices and with Low Energy Imaging. Australasian Physical and Engineering Science in Medicine, 39, 677-685. https://doi.org/10.1007/s13246-016-0463-9

[15] Zhang, M., Driewer, J., Zhang, Y., Zhou, S. and Zhu, X. (2015) The Measurement Accuracy of Ball Bearing Center in Portal Images Using an Intensity-Weighted Centroid Method. International Journal of Medical Physics, Clinical Engineering and Radiation Oncology, 4, 273-283. https://doi.org/10.4236/ijmpcero.2015.44033

[16] Zhang, M., Zhou, S.-M. and Qu, T. (2015) What Do We Mean When We Talk about the Linac Isocenter? International Journal of Medical Physics, Clinical Engineering and Radiation Oncology, 4, 233-242.

https://doi.org/10.4236/ijmpcero.2015.43028

[17] Sharpe, M.B., Moseley, D.J., Purdie, T.G., Islam, M., Siewerdsen, J.H. and Jaffray, D.A. (2006) The Stability of Mechanical Calibration for a kV Cone Beam Computed Tomography System Integrated with Linear Accelerator. Medical Physics, 33, 136-144. https://doi.org/10.1118/1.2143141

[18] Welzl, E. (1991) Smallest Enclosing Disks (Balls and Ellipsoids). New Results and New Trends in Computer Science, Graz, 20-21 June 1991, 359-370.

[19] Moyers, M.F. and Lesyna, W. (2004) Isocenter Characteristics of an External Ring Proton Gantry. International Journal of Radiation Oncology Biology Physics, 60, 1622-1630. https://doi.org/10.1016/j.ijrobp.2004.08.052

[20] Schiefer, H., Ingulfsen, N., Kluckert, J., Peters, S. and Plasswilm, L. (2015) Measurements of Isocenter Path Characteristics of the Gantry Rotation Axis with a Smartphone Application. Medical Physics, 42, 1184-1192. https://doi.org/10.1118/1.4906248

[21] Glide-Hurst, C., Bellon, M., Foster, R., et al. (2013) Commissioning of the Varian True Beam Linear Accelerator: A Multi-Institutional Study. Medical Physics, 40, Article ID: 031719. https://doi.org/10.1118/1.4790563

[22] Liu, G., van Doorn, T. and Bezak, E. (2004) The Linear Accelerator Mechanical and Radiation Isocentre Assessment with an Electronic Portal Imaging Device (EPID). Australasian Physics \& Engineering Sciences in Medicine, 27, 111-117. https://doi.org/10.1007/BF03178670

[23] Hyer, D.E., Mart, C.J. and Nixon, E. (2012) Development and Implementation of an EPID-Based Method for Localizing Isocenter. Journal of Applied Clinical Medical Physics, 13, 72-81. https://doi.org/10.1120/jacmp.v13i6.3965

[24] Nyflot, M.J., Cao, N., Meyer, J. and Ford, E.C. (2014) Improved Accuracy for Noncoplanar Radiotherapy: An EPID-Based Method for Submillimeter Alignment of Linear Accelerator Table Rotation with MV Isocenter. Journal of Applied Clinical Medical Physics, 15, 151-159. https://doi.org/10.1120/jacmp.v15i2.4682 